\title{
Synthesis and spectral characterization of some new thiazolopyrimidine derivatives
}

\author{
Mahmoud S. Tolba ${ }^{a^{*}}$, Mostafa Sayed ${ }^{a}$, Shaban A. A. Abdel-Raheem ${ }^{b}$, Taher A. Gaber ${ }^{c}$, Adel M. \\ Kamal El-Dean ${ }^{c}$ and Mostafa Ahmed ${ }^{a}$
}

${ }^{a}$ Chemistry Department, Faculty of Science, New Valley University, El-Kharja, 72511, Egypt

${ }^{b}$ Soil, Water, and Environment Research Institute, Agriculture Research Center, Giza, Egypt ${ }^{c}$ Chemistry Department, Faculty of Science, Assiut University, 71516 Assiut, Egypt

\section{H R O N I C L E}

Article history:

Received December 18, 2020

Received in revised form April 23, 2021

Accepted April 23, 2021

Available online

April 23, 2021

Keywords:

Pyrimidine

Thiazolopyrimidne

Synthesis

Spectral Characterization

\section{A B S T R A C T}

Thiazolopyrimidnes are considered one of the most interesting classes in heterocyclic chemistry due to their pharmaceutical importance. Herein, we report the synthesis of some new heterocyclic compounds containing thiazolopyrimidine starting from compound (1) which was previously prepared in literature. The starting compound was allowed to react with different alkylating agents such as chloroacetone, chloroacetyl chloride, and phenacyl bromide to afford derivatives (2-4). Compound (5), benzylidene derivative, was obtained by the reaction of compound (2) with benzaldehyde while amino-dicarbonitrile compound (6) can be obtained by the reaction of compound (5) and malononitrile. Acetylation of amino group in compound (6) with chloroacetyl chloride led to formation of compound (7). Nucleophilic substitution of chlorine in compound (7) by aniline gave compound (8) which further subjected the Munich reaction to form compound (9). All new synthesized compounds were characterized using different elemental and spectral analysis.

\section{Introduction}

Fused pyrimidines continue to attract considerable attention of researchers in different countries because of their great practical usefulness, primarily, due to a very wide spectrum of their biological activities. $^{1-3}$ Thiazolopyrimidine is one of the most interesting heterocyclic scaffolds possessing structural similarity to 5-fluorouracil (5-FU)-the well-known cancer metabolite. In addition, they have been reported to possess various important potent activities such as antimicrobial, antipsychotic, antiinflammatory, anti-Parkinson's, analgesic, antidepressant, anti-HIV, and anticancer activities. ${ }^{4-12}$ Besides, thiazolopyrimidine have been known with their bioactivities as transient receptor potential vanilloid-receptor 1 (TRPV1) modulators, ${ }^{13,14}$ antioxidants, ${ }^{15,16}$ pesticides, ${ }^{17}$ phosphate inhibitors, ${ }^{18,19}$ acetylcholinesterase inhibitors, ${ }^{20,21}$ and antimicrobial activities. ${ }^{22-24}$ Accordingly, and in continuation of our ongoing interest regarding the synthesis of biologically active molecules containing nitrogen and

* Corresponding author.

E-mail address: Mahmoud.Tolba@sci.nvu.edu.eg (M. S. Tolba)

(C) 2021 Growing Science Ltd. All rights reserved. doi: $10.5267 /$ j.ccl.2021.4.004 
sulphur heteroatom, ${ }^{25-51}$ we report here a convenient and efficient method for the synthesis of several novel derivatives including pyrimidine building block.

\section{Results and Discussion}

As shown in Fig. 1, in the alkylation of compound 1, which prepared according to reported procedure, ${ }^{52}$ with different alkylating agents such as chloroacetyl chloride, chloroacetone and phenacyl bromide, the alkylation of mercapto group was occurred to produce the $S$-alkylated products $\mathbf{2 - 4}$. The structure of the new obtained compounds was confirmed by the elemental and spectral analysis where the IR spectrum of compound 2 showed two $\mathrm{C}=\mathrm{O}$ groups $1663,1655 \mathrm{~cm}^{-1}$ while the ${ }^{1} \mathrm{H}$ NMR spectrum showed singlet signal at $4.37 \mathrm{ppm}$ characteristic for $\mathrm{CH}_{2}$ group. In addition, the ${ }^{1} \mathrm{H}$ NMR spectrum of compound 4 showed multiplet signals at $\mathbf{7 - 8} \mathrm{ppm}$, which proved the introduction of additional phenyl rings.

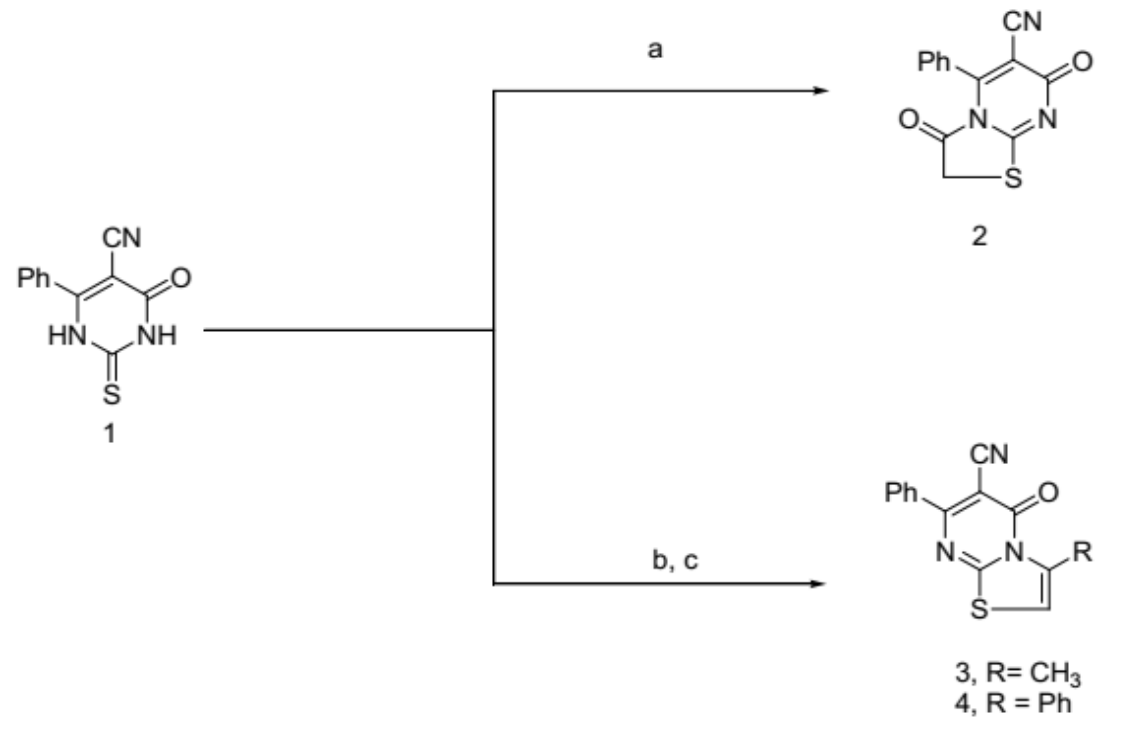

Reagents and condiation :(a) $\mathrm{ClCH}_{2} \mathrm{COCl}$, dioxane, TEA, reflux 6h; (b) $\mathrm{ClCH}_{2} \mathrm{COCH}_{3}, \mathrm{CH}_{3} \mathrm{COONa}$, EtOH, reflux 4h.; (c) $\mathrm{PhCOCH}_{2} \mathrm{Br}, \mathrm{CH}_{3} \mathrm{COONa}$, EtOH, 4h.

Fig. 1. Synthesis of compounds 2-4.

As shown in Fig. 2, condensation of compound $\mathbf{2}$ with benzaldehyde gave a cyclic chalcone derivative 5 which in turn reacted with malononitrile to give the amino-dicarbonitrile compound $\mathbf{6}$. After that, acetylation of the amino group in compound $\mathbf{6}$ with chloroacetyl chloride led to the formation of 2-Chloro-N-(3,8-dicyano-7-oxo-4,9-diphenyl-5a,6-dihydro-4H,7H-pyrano[2',3':4,5]thiazolo[3,2a]pyrimidin-2-yl)acetamide compound (7). When the latter compound was allowed to react with aniline, nucleophilic substitution occurred to produce compound $\mathbf{8}$, which reacted with formaldehyde under Munich condition to give compound 9. The structure of the obtained compounds was easily confirmed by the spectral analysis where the ${ }^{1} \mathrm{H}$ NMR spectrum of compound $\mathbf{5}$ showed two singlet signals at 3.88 and $5.93 \mathrm{ppm}$ characteristic for $\mathrm{CH}=\mathrm{CH}$ protons as well as increasing the aromatic protons by $5 \mathrm{H}$. Also, the IR spectrum of compound 6 showed the appearance of two bands at 3321 , $3245 \mathrm{~cm}^{-1}$ characteristics for amino groups whereas the ${ }^{1} \mathrm{H}$ NMR spectrum exhibited singlet signal at $6.64 \mathrm{ppm}$ characteristics for $\mathrm{NH}_{2}$ group. Finally, the structure of Munich product 9 was proved by the spectral analysis, where its ${ }^{1} \mathrm{H}$ NMR spectrum showed two singlet signals at 4.22 and $4.56 \mathrm{ppm}$ characteristics for $2 \mathrm{CH}_{2}$ groups. 

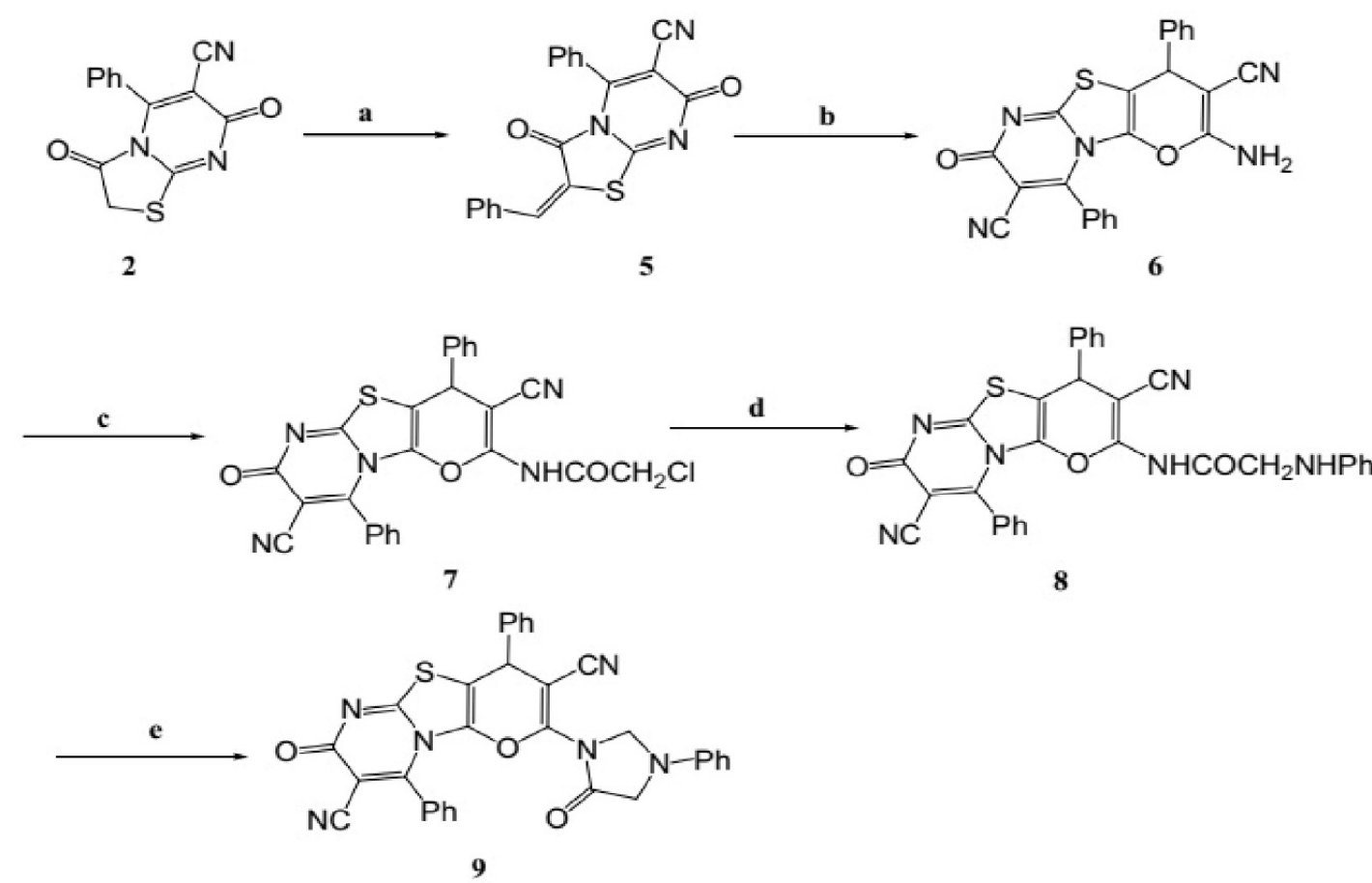

Reagents and conditions: (a) PhCHO, Piperidine, $\mathrm{EtOH}$; (b) $\mathrm{CH}_{2}(\mathrm{CN})_{2}$, Piperidine; (c) $\mathrm{ClCH}_{2} \mathrm{COCl}$, dioxane; (d) $\mathrm{PhNH}_{2}, \mathrm{EtOH}$; (e) $\mathrm{HCHO}$, EtOH.

Fig. 2. Synthesis of compounds 5-9

\section{Conclusion}

In the present work, we introduced a synthetic route for the synthesis of some new heterocyclic compounds containing thiazolopyrimidine moiety. Since, the compound 4-oxo-6-phenyl-2-thioxo1,2,3,4-tetrahydropyrimidine-5-carbonitrile (1) was prepared according to the reported procedure and used as a precursor for synthesizing the target compounds. In addition, all the synthesized compounds were characterized using spectroscopic data and elemental analyses.

\section{Experimental}

\subsection{Materials and methods}

All melting points are uncorrected and measured on a Fisher-John apparatus. Elemental analysis was determined on an elementar analysensysteme GmbH - vario EL III elemental analyzer in the central lab of Assiut University. Their results were found to be in good agreement $( \pm 0.2 \%)$ with the calculated values. IR spectra were recorded on a Pye-Unicam Sp-100 spectrophotometer using $\mathrm{KBr}$ wafer technique. ${ }^{1} \mathrm{H}$ NMR spectra were obtained on BRUKER $400 \mathrm{MHz}$ spectrometers in a suitable deuterated solvent using tetramethylsilane as an internal standard (chemical shifts in ppm), otherwise stated. Mass spectra were obtained on JEOL JMS-600 apparatus. Preparative and analytical TLC were carried out on silica gel plates (Fluka 70643-50EA. SIGMA-ALDRICH, Germany) using UV light All reactions were carried out under an air atmosphere. 4-oxo-6-phenyl-2-thioxo-1,2,3,4-tetrahydropyrimidine-5carbonitrile (1) was prepared according to the reported procedure. ${ }^{52}$

\subsection{Synthetic procedure for 3,7-Dioxo-5-phenyl-2,3-dihydro-7H-thiazolo[3,2-a]pyrimidine-6- carbonitrile (2)}


To a solution of compound (1) $(0.75 \mathrm{~g}, 3 \mathrm{mmol})$ in dioxane $(10 \mathrm{~mL})$ containing triethyl amine $(0.3$ $\mathrm{mL}, 3 \mathrm{mmol})$, chloroacetyl chloride $(0.38 \mathrm{~mL}, 3 \mathrm{mmol})$ was added in small portions with continuous cooling and stirring, the mixture was heated at reflux in a water bath for $6 \mathrm{~h}$. The solid obtained was filtered off and recrystallized from benzene as orange crystals in $66 \%$ yield, m.p.268-270 ${ }^{\circ} \mathrm{C}$. FT-IR $(\mathrm{KBr}), v\left(\mathrm{~cm}^{-1}\right), 3040\left(\mathrm{CH}\right.$ aromatic) 1663, $1655(2 \mathrm{C}=\mathrm{O}), 2204(\mathrm{CN}) .{ }^{1} \mathrm{H}$ NMR $(300 \mathrm{MHz}, \mathrm{DMSO}-\mathrm{d} 6)$ $\delta$ (ppm): $4.37\left(\mathrm{~s}, 2 \mathrm{H}, \mathrm{CH}_{2}\right), 8.55(\mathrm{~m}, 5 \mathrm{H}, \mathrm{Ar}-\mathrm{H})$. Elemental Analysis Calculated for $\mathrm{C}_{13} \mathrm{H}_{7} \mathrm{~N}_{3} \mathrm{O}_{2} \mathrm{~S}(\%)$ C, 57.99; H, 2.62; N, 15.61; S, 11.91. Found (\%): C, 57.77; H, 2.70; N, 15.57; S, 11.88.

\subsection{Synthetic procedure for 3-Methyl-5-oxo-7-phenyl-5H-thiazolo[3,2-a]pyrimidine-6-carbonitrile (3).}

A mixture of compound (1) $(0.50 \mathrm{~g}, 2 \mathrm{mmol})$, chloroacetone $(0.2 \mathrm{ml}, 2 \mathrm{mmol})$ and fused sodium acetate $(0.49 \mathrm{~g}, 5 \mathrm{mmol})$ were added to ethanol, the resultant mixture was heated at reflux for $4 \mathrm{~h}$. the sodium salt obtained after cooling was filtered off and dissolved in water then neutralized with $\mathrm{HCl}$ to give reddish brown precipitate then filtered off, dried and recrystallized from ethanol: dioxane mixture $(1: 1)$ as pale brown crystals in $72 \%$ yield, m.p.302-304 ${ }^{\circ} \mathrm{C}$. FT-IR $(\mathrm{KBr}), v\left(\mathrm{~cm}^{-1}\right): 3056(\mathrm{CH}$ aromatic), 2936 (CH aliphatic), $1655(\mathrm{C}=\mathrm{O}), 2207(\mathrm{CN}) .{ }^{1} \mathrm{H}$ NMR $\left(300 \mathrm{MHz}, \mathrm{DMSO}-\mathrm{d}_{6}\right) \delta(\mathrm{ppm}): 2.67(\mathrm{~s}, 3 \mathrm{H}$, $\left.\mathrm{CH}_{3}\right), 6.73(\mathrm{~s}, 1 \mathrm{H}, \mathrm{CH}), 7.16-8.45(\mathrm{~m}, 5 \mathrm{H}, \mathrm{Ar}-\mathrm{H}) .{ }^{13} \mathrm{C}$ NMR $\left(75 \mathrm{MHz}, \mathrm{DMSO}-\mathrm{d}_{6}\right) \delta(\mathrm{ppm}): 16.35$, 104.66, 111.75, 118.76, 124.63, 127.67, 128.56, 134.45, 143.55, 156.67, 160.32, 165.34. Elemental Analysis Calculated for $\mathrm{C}_{14} \mathrm{H}_{9} \mathrm{~N}_{3} \mathrm{OS}(\%) \mathrm{C}, 62.91 ; \mathrm{H}, 3.39 ; \mathrm{N}, 15.72 ; \mathrm{S}, 11.99 \%$ Found (\%) C, 62.87; H, 3.35; N, 15.76; S, 11.96 .

\subsection{Synthetic procedure for 5-Oxo-3,7-diphenyl-5H-thiazolo[3,2-a]pyrimidine-6-carbonitrile (4).}

A mixture of compound (1) $(0.50 \mathrm{~g}, 2 \mathrm{mmol})$, phenacyl bromide $(0.4 \mathrm{~g}, 2 \mathrm{mmol})$ and fused sodium acetate $(0.4 \mathrm{~g}, 5 \mathrm{mmol})$ were added to ethanol, the resultant mixture was refluxed for $4 \mathrm{~h}$. The reaction mixture was cooled and poured into water solution. The solid obtained after neutralization with $\mathrm{HCl}$ was filtered off, dried and crystallized from ethanol:dioxane $(2: 1)$ as pale yellow crystals in $88 \%$ yield, m.p.322-324 ${ }^{\circ}$ C. FT-IR (KBr), v $\left(\mathrm{cm}^{-1}\right)$ : $3045\left(\mathrm{CH}\right.$ aromatic), $1664(\mathrm{C}=\mathrm{O}), 2213(\mathrm{CN}) .{ }^{1} \mathrm{H}$ NMR $(300$ MHz, DMSO-d 6 ) $\delta(\mathrm{ppm}): 6.65(\mathrm{~s}, 1 \mathrm{H}, \mathrm{CH}), 7.15-8.67(\mathrm{~m}, 10 \mathrm{H}, \mathrm{Ar}-\mathrm{H}) .{ }^{13} \mathrm{C}$ NMR $(75 \mathrm{MHz}, \mathrm{DMSO}-$ d6) $\delta$ (ppm): 102.34, 109.63, 117.23, 126.55, 127.16, 127.96, 128.45, 128.87, 129.17, 129.46, 129.68, 137.65, 143.31, 158.56 165.4,168.66. Elemental Analysis Calculated for $\mathrm{C}_{19} \mathrm{H}_{11} \mathrm{~N}_{3} \mathrm{OS}(\%) \mathrm{C}, 69.29 ; \mathrm{H}$, 3.37 ; N, 12.76; S, $9.73 \%$ Found (\%) C, 69.33; H, 3.34; N, 12.72; S, 9.76.

4.5 Synthetic procedure for (E)-2-Benzylidene-3,7-dioxo-5-phenyl-2,3-dihydro-7H-thiazolo[3,2a]pyrimidine-6-carbonitrile (5).

A mixture of compound (2) $(0.50 \mathrm{~g}, 1.8 \mathrm{mmol})$, benzaldehyde $(2 \mathrm{mmol})$ and piperidine $(2 \mathrm{~mL})$ were added to ethanol, the resultant mixture was refluxed for $5 \mathrm{~h}$, The solid obtained during heating was collected, filtered off, dried and crystallized from ethanol: dioxane (2:1) as yellow crystals in 74\% yield, m.p.277-279 ${ }^{\circ} \mathrm{C}$. FT-IR $(\mathrm{KBr}), v\left(\mathrm{~cm}^{-1}\right): 3042(\mathrm{CH}$ aromatic), $1678(\mathrm{C}=\mathrm{O}), 1664(\mathrm{C}=\mathrm{O}), 2213$ (CN). ${ }^{1} \mathrm{H}$ NMR $\left(300 \mathrm{MHz}, \mathrm{DMSO}-\mathrm{d}_{6}\right) \delta(\mathrm{ppm}): 7.16-8.68$ (m, $\left.10 \mathrm{H}, \mathrm{Ar}-\mathrm{H}\right), 9.45$ (s, 1H, CH). ${ }^{13} \mathrm{C}$ NMR (75 MHz, DMSO-d6) $\delta$ (ppm): 79.65, 83.81, 116.74, 123.87, 127.98, 128.16, 128.56, 128.98, 129.65, 129.87, 131.13, 132.76, 136.45, 138.78, 158.15, 164.18, 168.32. Elemental Analysis Calculated for $\mathrm{C}_{20} \mathrm{H}_{11} \mathrm{~N}_{3} \mathrm{O}_{2} \mathrm{~S}(\%)$ C, 67.22; H, 3.10; N, 11.76; S, 8.97 Found (\%): C, 67.31; H, 3.01; N, 11.64; S, 9.02.

4.6 Synthetic procedure for 2-Amino-7-oxo-4,9-diphenyl-4H,7H-pyrano[2',3':4,5]thiazolo[3,2a]pyrimidine-3,8-dicarbonitrile (6).

A mixture of compound (5) $(0.45 \mathrm{~g}, 1.25 \mathrm{mmol})$, malononitrile $(0.3 \mathrm{~g}, 4.54 \mathrm{mmol})$ and piperidine (1 $\mathrm{mL}$ ) were added to ethanol, the resultant mixture was heated under reflux for $3 \mathrm{~h}$. The solid obtained after cooling was collected, filtered off, dried and crystallized from dioxane brown crystals in $46 \%$ yield, m.p.302-304 ${ }^{\circ} \mathrm{C}$. FT-IR (KBr), v $\left(\mathrm{cm}^{-1}\right): 3321,3245\left(\mathrm{NH}_{2}, \mathrm{NH}\right), 3052$ (CH aromatic), 1669 $(\mathrm{C}=\mathrm{O}), 2213,2204(2 \mathrm{CN}) .{ }^{1} \mathrm{H}$ NMR $\left(300 \mathrm{MHz}, \mathrm{DMSO}-\mathrm{d}_{6}\right) \delta(\mathrm{ppm}): 5.93(\mathrm{~s}, 1 \mathrm{H}, \mathrm{CH}), 6.64(\mathrm{~s}, 2 \mathrm{H}$, $\left.\mathrm{NH}_{2}\right), 7.12-8.89$ (m, $\left.10 \mathrm{H}, \mathrm{Ar}-\mathrm{H}\right) .{ }^{13} \mathrm{C}$ NMR (75 MHz, DMSO-d6) $\delta$ (ppm): 72.55, 77.78, 85.45, 89.49, 
$117.57,118.22,123.26,124.55,128.01,128.45,128.98,129,44,129.67,129.81,130.15,132,55$, 136,23, 141.32, 148.45, 157,56, 165,33. Elemental Analysis Calculated for $\mathrm{C}_{23} \mathrm{H}_{13} \mathrm{~N}_{5} \mathrm{O}_{2} \mathrm{~S}(\%) \mathrm{C}, 65.24$; H, 3.09; N, 16.54; S, 7.57 Found (\%): C, 65.32; H, 2.99; N, 16.62; S, 7.43.

4.7 Synthetic procedure for 2-Chloro-N-(3,8-dicyano-7-oxo-4,9-diphenyl-4H,7Hpyrano[2',3':4,5]thiazolo[3,2-a]pyrimidin-2-yl)acetamide (7).

A mixture compound $6(0.75 \mathrm{~g}, 1.7 \mathrm{mmol})$, and chloroacetylchloride $(0.45 \mathrm{~mL}, 3.9 \mathrm{mmol})$ in dioxane $(20 \mathrm{~mL})$ was heated on steam bath for 3 hours. After cooling, the mixture was poured onto water and neutralized with diluted sodium carbonate solution The solid thus formed was filtered, washed with water, dried and recrystallized from ethanol-water (2:1) to afford reddish brown crystals in $56 \%$ yield; m.p. $268-270^{\circ} \mathrm{C}$. FT-IR (KBr), v $\left(\mathrm{cm}^{-1}\right)$ : $3250(2 \mathrm{NH}), 3055$ (C-H aromatic), 2925, 2866 (C-H aliphatic), 2214, 2204 (2CN), 1668, 1678 (2CO amidic). ${ }^{1} \mathrm{H}$ NMR (300 MHz, DMSO-d6): 4.27 (s, 2H, $\left.\mathrm{CH}_{2}\right), 5.88(\mathrm{~s}, 1 \mathrm{H}, \mathrm{CH}), 7.14-8.76(\mathrm{~m}, 10 \mathrm{H}, \mathrm{Ar}-\mathrm{H}), 9.43(\mathrm{~s}, 1 \mathrm{H}, \mathrm{NH})$. Elemental Analysis Calculated for $\mathrm{C}_{25} \mathrm{H}_{14} \mathrm{ClN}_{5} \mathrm{O}_{3} \mathrm{~S}(\%) \mathrm{C}, 60.06 ; \mathrm{H}, 2.82 ; \mathrm{N}, 14.01 ; \mathrm{S}, 6.41$ Found $(\%)$ : C, 60.11; H, 2.90; N, 14.01; $\mathrm{S}, 6.21$.

4.8 Synthetic procedure for N-(3,8-Dicyano-7-oxo-4,9-diphenyl-4H,7H-pyrano[2',3':4,5]thiazolo[3,2a]pyrimidin-2-yl)-2-(phenylamino)acetamide (8).

A mixture of chloroacetamide derivative $7(0.5 \mathrm{~g}, 0.99 \mathrm{mmol})$ and aniline $(2 \mathrm{mmol})$ was dissolved in $20 \mathrm{~mL}$ ethanol and refluxed for $3 \mathrm{~h}$. The solid precipitate, which was formed, filtered off, dried and recrystallized from ethanol-water $(2: 1)$ to give pale brown needles in $46 \%$ yield; m.p. $315-317^{\circ} \mathrm{C}$. FTIR (KBr), v ( $\left.\mathrm{cm}^{-1}\right):$ 3265, 3167 (2NH), 3053 (C-H aromatic), 2913, 2834 (C-H aliphatic), 2217, 2203 $(2 \mathrm{CN}), 1666,1679$ (2CO amidic). ${ }^{1} \mathrm{H}$ NMR (300 MHz, DMSO-d $): 4.27\left(\mathrm{~s}, 2 \mathrm{H}, \mathrm{CH}_{2}\right), 5.11(\mathrm{~s}, 1 \mathrm{H}$, $\mathrm{NH}), 5.88(\mathrm{~s}, 1 \mathrm{H}, \mathrm{CH}), 7.16-8.87(\mathrm{~m}, 15 \mathrm{H}, \mathrm{Ar}-\mathrm{H}), 8.55(\mathrm{~s}, 1 \mathrm{H}, \mathrm{NH})$. Elemental Analysis Calculated for $\mathrm{C}_{31} \mathrm{H}_{20} \mathrm{~N}_{6} \mathrm{O}_{3} \mathrm{~S}(\%) \mathrm{C}, 66.90 ; \mathrm{H}, 3.62 ; \mathrm{N}, 15.10 ; \mathrm{S}, 5.76$. Found $(\%)$ : C, 66.95; H, 3.54; N, 15.14; $\mathrm{S}$, 5.73 .

4.9 Synthetic procedure for 7-Oxo-2-(5-oxo-3-phenylimidazolidin-1-yl)-4,9-diphenyl-4H,7Hpyrano[2',3':4,5]thiazolo[3,2-a]pyrimidine-3,8-dicarbonitrile (9).

To a solution of compounds $8(0.25 \mathrm{~g}, 0.44 \mathrm{mmol})$ in ethanol $(10 \mathrm{ml})$ a few drops of formaldehyde $(1.2 \mathrm{~mL})$ was added. The solution was refluxed for $4 \mathrm{hrs}$. The solid obtained on hot, filtered off, dried and recrystallized from ethanol: dioxane $(2: 1)$ to give brown precipitate in $52 \%$ yield; m.p. $342-344^{\circ} \mathrm{C}$. FT-IR (KBr), v $\left(\mathrm{cm}^{-1}\right): 3265(\mathrm{NH}), 3054$ (C-H aromatic), 2977 (C-H aliphatic), 2213, $2205(2 \mathrm{CN})$, 1663, 1671 (CO amidic). ${ }^{1} \mathrm{H}$ NMR (300 MHz, DMSO-d6): 4.22 (s, 2H, $\left.\mathrm{CH}_{2}\right), 4.56$ (s, 2H, $\left.\mathrm{CH}_{2}\right), 6.01$ (s, $1 \mathrm{H}, \mathrm{CH}), 7.12-8.76$ (m, $15 \mathrm{H}, \mathrm{Ar}-\mathrm{H})$. Elemental Analysis Calculated for $\mathrm{C}_{32} \mathrm{H}_{20} \mathrm{~N}_{6} \mathrm{O}_{3} \mathrm{~S}(\%) \mathrm{C}, 67.59$; H, 3.55; N, 14.78; S, 5.64. Found (\%): C, 67.65; H, 3.45; N, 14.85; S, 5.57.

\section{References}

1 Al-Harbi N. O., Bahashwan S. A., Fayed A. A., Aboonq M. S., and Amr A. E. G. (2013) Antiparkinsonism, hypoglycemic and anti-microbial activities of new poly fused ring heterocyclic candidates. Int. J. Biol. Macromol., 57 (2013) 165-73.

2 Sukach V. A., Tkachuk V. M., Rusanov E. B., Roschenthaler G. V., and Vovk M. V. (2012) Heterocyclization of $\mathrm{N}$-(1-chloro-2,2,2-trifluoro ethylidene)carbamates with $\beta$-enamino esters-a novel synthetic strategy to functionalized trifluoromethylated pyrimidines. Tetrahedron, 68 (40) 8408-15.

3 Gondkar A. S., Deshmukh V. K., and Chaudhari S. R. (2013) Synthesis, characterization and in vitro antiinflammatory activity of some substituted 1,2,3,4 tetrahydropyrimidine derivatives. Drug. invent. Today, 5 (3) 175-81. 
4 Mohamed A. M., Amr A. E. G., Alsharari M. A., Al-Qalawi H. R. M., Germoush M. O., and AlOmar M. A. (2011) Anticancer activities of some new synthesized thiazolo[3,2-a]pyrido[4,3d]pyrimidine derivatives. Am. J. Biochem. Biotechnol., 7 (2) $43-54$.

5 Hammam A. G., El-Salam O. I. A., Mohamed A. M., and Hafez N. A. (2005) Novel fluoro substituted benzo[o]pyran with anti-lung cancer activity. Ind. J. Chem., 44 (9) 1887-1893.

6 Flefel E. E., Salama M. A., El-Shahat M., El-Hashash M. A., and El-Farargy A. F. (2007) A novel synthesis of some new pyrimidine and thiazolopyrimidine derivatives for anticancer evaluation. Phosphorus Sulfur Silicon Relat.Elem., 182 (8) 1739-1756.

7 Cai D., Zhang Z.-H., Chen Y., Yan X.-J., Zou L.-J., Wang Y.-X., and Liu X.-Q. (2015) Synthesis, antibacterial and antitubercular activities of some $5 \mathrm{H}$-thiazolo[3,2-a]pyrimidin-5-ones and sulfonic acid derivative. Molecules, 20 (9) 16419-16434.

8 Wichmann J., Adam G., Kolczewski S., Mutel V., and Woltering T. (1999) Structure-activity relationships of substituted $5 \mathrm{H}$-thiazolo[3,2-a]pyrimidines as group 2 metabotropic glutamate receptor antagonists. Bioorg. Med. Chem. Lett., 9 (11) 1573-1576.

9 Al-Omary F. A., Hassan G. S., El-Messery S. M., and ElSubbagh H. I. (2012) Substituted thiazoles V. Synthesis and antitumor activity of novel thiazolo[2,3-b]quinazoline and pyrido[4,3-d] thiazolo[3,2-a] pyrimidine analogues. Eur. J. Med. Chem., 47 (2012) 65-72.

10 Fatima S., Sharma A., Saxena R., Tripathi R., Shukla S. K., Pandey S. K., Tripathi R., and Tripathi R. P. (2012) One pot efficient diversity oriented synthesis of polyfunctional styryl thiazolopyrimidines and their bio-evaluation as antimalarial and anti-HIV agents. Eur. J. Med. Chem., 55 (2012) 195-204.

11 Yıldırım A. B., Mutlu E., and Yıldırım M. (2018) Cytotoxic Effects of Thiazolo[3,2-C]Pyrimidines Against Mcf-7 And Hepg2/C3a Carcinoma Cell Lines. Hacet. J. Biol. Chem., 2 (46) 237-246.

12 Amr A.-E.-G., Maigali S. S., and Abdulla M. M. (2008) Synthesis, and analgesic and antiparkinsonian activities of thiopyrimidine, pyrane, pyrazoline, and thiazolopyrimidine derivatives from 2-chloro-6-ethoxy-4-acetylpyridine. Mon. Chem., 139 (11) 1409-1415.

13 Lebsack A. D., Branstetter B. J., Hack M. D., Xiao W., Peterson M. L., Nasser N., Maher M. P., Hong A., Bhattacharya A., Kansagara M., Scott B. P., Luo L., Rynberg R., Rizzolio M., and Sandr R. (2009) Identification and synthesis of 2,7-diamino-thiazolo[5,4- $d$ ]pyrimidine derivatives as TRPV1 antagonists. Bioorganic Med. Chem. Lett., 19 (1) 40-46.

14 Said M., Abouzid K., Mouneer A., Ahmedy A., and Osman A.-M. (2004) Synthesis and biological evaluation of new thiazolopyrimidines. Arch. Pharm. Res., 27 (5) 471-477.

15 Ackova D. G., Kotur-Stevuljevic J., Mishra C. B., Luthra P. M., and Saso L. (2019) Antioxidant Properties of Synthesized Bicyclic Thiazolopyrimidine Derivatives as Possible Therapeutic Agents. Appl. Sci., 9 (1) 113-122.

16 Youssef M. M., and Amin M. A. (2012) Microwave Assisted Synthesis of Some New Thiazolopyrimidine, Thiazolodipyrimidine and Thiazolopyrimidothiazolopyrimidine Derivatives with Potential Antioxidant and Antimicrobial Activity. Molecules, 17 (8) 9652-9667.

17 Linder W., and Brandes W. (1991) Pesticidal Thiazolopyrimidine Derivatives. World Patent Information, 13 (4) 206-208.

18 Duval R., Kolb S., Braud E., Genest D., and Garbay C. (2009) Rapid discovery of triazolobenzylidenethiazolopyrimidines (TBTP) as CDC25 phosphatase inhibitors by parallel click chemistry and in situ screening. J. Comb. Chem., 11 (6) 947-950.

19 Kolb S., Mondésert O., Goddard M. L., Jullien D., Villoutreix B. O., Ducommun B., Garbay C., and Braud E. (2009) Development of novel thiazolopyrimidines as CDC25B phosphatase inhibitors. Chem Med Chem, 4 (4) 633-648.

20 Mahgoub M. Y., Elmaghraby A. M., Harb A. A., da Silva J. L. F., Justino G. C., and Marques M. M.( 2019) Synthesis, crystal structure, and biological evaluation of fused thiazolo[3,2-a]pyrimidines as new acetylcholinesterase inhibitors. Molecules, 24 (12) 2306-2335.

21 Liu S.-J., Yang L., Jin Z., Huang E. F., Wan D. C. C., Lin H.-Q., and Hu C. (2009) Design, synthesis, and biological evaluation of $7 \mathrm{H}$-thiazolo[3,2-b]- 1,2,4-triazin-7-one derivatives as novel acetylcholinesterase inhibitors. Arkivoc, 2009 (10) 333-348. 
22 Rashad A. E., Shamroukh A. H., Abdel-Megeid R. E., and El-Sayed W. A. (2010) Synthesis, reactions and antimicrobial evaluation of some polycondensedthieno-pyrimidine derivatives. Synth. Commun., 40 (8) 1149-1160.

23 El-Emary T. I., and Abdel-Mohsen S. A. (2006) Synthesis and antimicrobial activity of some new 1,3-diphenylpyrazoles bearing pyrimidine, Pyrimidinethione, thiazolopyrimidine, triazolopyrimidine, thio- and alkylthiotriazolopyrimidinone moieties at the 4-position. Phosphorus Sulfur Silicon Relat. Elem., 181 (11) 2459-2474.

24 Maddila S., Damu G. L.V., Oseghe E. O., Abafe O. A., Venakata R. C., and Lavanya P. (2012) Synthesis and biological studies of novel biphenyl-3,5-dihydro-2H-thiazolo-pyrimidines derivatives. J. Korean Chem. Soc., 56 (3) 334-340.

25 Kamal El-Dean A. M., Zaki R. M., and Abdulrazzaq A. Y. (2015) A Convenient Synthesis and Biological Activity of Novel Thieno[2,3-c]Pyrazole Compounds as Antimicrobial and AntiInflammatory Agents. Russ. J. Bioorg. Chem., 41 (1) 97-104.

26 Saddik A. A., Kamal El-Dean A. M., El-Said W. A., Hassan K. M., and Abbady M. S. (2018) Synthesis, Antimicrobial, and Anticancer Activities of a New Series of Thieno[2,3-d] Pyrimidine Derivatives. J. Heterocyclic Chem., 55 (9) 2111-2122.

27 Kamal El Dean A. M., Zaki R. M., Geies A. A., Radwan S. M., and Tolba M. S. (2013) Synthesis and Antimicrobial Activity of New Heterocyclic Compounds Containing Thieno[3,2-c]Coumarin and Pyrazolo[4,3-c]Coumarin Frameworks. Russ. J. Bioorganic Chem., 39 (5) 553-564.

28 Saber A. F., Sayed M., Tolba M. S., Kamal A. M., Hassanien R., and Ahmed M. (2021) A Facile Method for Preparation and Evaluation of the Antimicrobial Efficiency of Various Heterocycles Containing Thieno[2,3-d]Pyrimidine. Synth. Commun, 51 (3) 398-409.

29 Zaki R. M., Kamal El Dean A. M., Radwan S. M., and Abdul-Malik M. A. (2018) A Convenient Synthesis, Reactions and Biological Activities of Some Novel Thieno[3,2-e]Pyrazolo[3,4b]Pyrazine Compounds as Anti-Microbial and Antiinflammatory Agents. Curr. Organic Synth., 15 (6) 863-871.

30 Ahmed M., Younis O., Orabi E. A., Sayed A. M., Kamal El-Dean A. M., Hassanien R., Davis R. L., Tsutsumi O., and Tolba M. S. (2020) Synthesis of novel thienopyrimidines as biocompatible chromophores with aggregation-induced emission sensitive to molecular aggregation. ACS Omega, 5 (46) 29988-30000.

31 Ahmed M., Sayed M., Saber A. F., Hassanien R., Kamal El-Dean A. M., and Tolba M. S. (2020) Synthesis, Characterization, and Antimicrobial Activity of New Thienopyrimidine Derivatives. Polycycl. Aromat. Compd., Accepted Manuscript (DOI: 10.1080/10406638.2020.1852587).

32 Kamal A. M., Radwan S. M., and Zaki R. M. (2011) Synthesis and Biological Activity of Pyrazolothienotetrahydroisoquinoline and $[1,2,4]$ Triazolo[3,4-a]ThienotetrahydroIsoquinoline Derivatives. Eur. J. Med. Chem., 46 (2) 567-578.

33 Sayed M., Kamal El-Dean A. M., Ahmed M., and Hassanien R. (2018) Synthesis of Some Heterocyclic Compounds Derived from Indole as Antimicrobial Agents. Synth. Commun., 48 (4) 413-421.

34 Tolba M. S., Kamal El-Dean A. M., Ahmed M., Hassanien R., and Farouk M. (2017) Synthesis and Antimicrobial Activity of Some New Thienopyrimidine Derivatives. Arkivoc, 2017 (5) 229-243.

35 Tolba M. S., Ahmed M., Kamal El-Dean A. M., Hassanien R., and Farouk M. (2018) Synthesis of new fused thienopyrimidines derivatives as anti-inflammatory agents. J. Heterocyclic Chem., 55 (2) $408-418$.

36 Sayed M., Younis O., Hassanien R., Ahmed M., Mohammed A. A. K., Kamal A. M., and Tsutsumi O. (2019) Design and synthesis of novel indole derivatives with aggregation-induced emission and antimicrobial Activity. J. Photochem. Photobiol. A., 383 (2019) 111969-111979.

37 Younis O., Tolba M. S., Orabi E. A., Kamal A. M., Hassanien R., Tsutsumi O., and Ahmed M. (2020) Biologically-active heterocyclic molecules with aggregation-induced blue-shifted emission and efficient luminescence both in solution and solid states. J. Photochem. Photobiol. A., 400 (2020) 112642-112653. 
38 Kamal El-Dean A. M., Zaki R. M., Radwan S. M., and Saber A. F. (2017) Synthesis, Reactions and Spectral Characterization of Novel Thienopyrazole Derivatives. Eur. Chem. Bull., 6 (12) 550-553.

39 Zaki R. M., Kamal El-Dean A. M., Radwan S. M., and Saber A. F. (2019) Efficient synthesis, reactions and spectral characterization of novel pyrazolo[4',3':4,5]thieno[3,2-d]pyrimidine derivatives and their related heterocycles. Heterocycl. Commun., 25 (1) 39-46.

40 Saber A. F., Zaki R. M., Kamal El-Dean A. M., and Radwan S. M. (2020) Synthesis, reactions and spectral characterization of some new biologically active compounds derived from thieno[2,3c]pyrazole-5-carboxamide. J. Heterocyclic Chem., 57 (1) 238-247.

41 Tolba M. S., Kamal El-Dean A. M., Ahmed M., and Hassanien R. (2019) Synthesis, reactions, and biological study of some new thienopyrimidine derivatives as antimicrobial and antiInflammatory agents. J. Chin. Chem. Soc., 66 (5) 548-557.

42 Bakhite E. A., Abd-Ella A. A., El-Sayed M. E. A., and Abdel-Raheem Sh. A. A. (2014) Pyridine derivatives as insecticides. Part 1: Synthesis and toxicity of some pyridine derivatives against Cowpea Aphid, Aphis craccivora Koch (Homoptera: Aphididae). J. Agric. Food Chem., 62 (41) 9982-9986.

43 Bakhite E. A., Abd-Ella A. A., El-Sayed M. E. A., and Abdel-Raheem Sh. A. A. (2017) Pyridine derivatives as insecticides. Part 2: Synthesis of some piperidinium and morpholinium cyanopyridinethiolates and their Insecticidal Activity. J. Saud. Chem. Soc., 21 (1) 95-104.

44 Kamal El-Dean A. M., Abd-Ella A. A., Hassanien R., El-Sayed M. E. A., Zaki R. M., and AbdelRaheem Sh. A. A. (2019) Chemical design and toxicity evaluation of new pyrimidothienotetrahydroisoquinolines as potential insecticidal agents. Toxicol. Rep., 6 (2019) 100104.

45 Saddik A. A., Hassan K. M., Kamal El-Dean A. M., and Abbady M. S. (2015) Synthesis of new mercaptopyrimidines and thienopyrimidines. Eur. Chem. Bull., 4 (9) 436-441.

46 Kamal El-Dean A. M., Abd-Ella A. A., Hassanien R., El-Sayed M. E. A., and Abdel-Raheem Sh. A. A. (2019) Design, Synthesis, Characterization, and Insecticidal Bioefficacy Screening of Some New Pyridine Derivatives. ACS Omega, 4 (5) 8406-8412.

47 Abdel-Raheem Sh. A. A., Kamal El-Dean A. M., Zaki R. M., Hassanien R., El-Sayed M. E. A., Sayed M., and Abd-Ella A. A. (2021) Synthesis and toxicological studies on distyryl-substituted heterocyclic insecticides. Eur. Chem. Bull., 10 (4) 225-229.

48 Sayed M., Kamal El-Dean A. M., Ahmed M., and Hassanien R. (2017) Synthesis of some new heterocyclic compounds containing indole moiety. Eur. Chem. Bull., 6 (4) 171-176.

49 Abdel-Raheem Sh. A. A., Kamal El-Dean A. M., Hassanien R., El-Sayed M. E. A., and Abd-Ella A. A. (2021) Synthesis and characterization of some distyryl-derivatives for agricultural uses. Eur. Chem. Bull., 10 (1) 35-38.

50 Abdel-Raheem Sh. A. A., Kamal El-Dean A. M., Hassanien R., El-Sayed M. E. A., and Abd-Ella A. A. (2020) Synthesis and biological activity of 2-((3-Cyano-4,6-distyrylpyridin-2-yl)thio)acetamide and its cyclized form. Alger. j. biosciences, 01 (02) 046-050.

51 Abdel-Raheem Sh. A. A., Kamal El-Dean A. M., Hassanien R., El-Sayed M. E. A., Sayed M., and Abd-Ella A. A. (2021) Synthesis and spectral characterization of selective pyridine compounds as bioactive agents. Curr. Chem. Lett., 10 (2021) 255-260.

52 Kambe S., Saito K., Kishi H., Sakurai A., and Midorikawa H. (1979) A One-Step Synthesis of 4Oxo-2-thioxopyrimidine Derivatives by the Ternary Condensation of Ethyl Cyanoacetate, Aldehydes, and Thiourea. Synthesis, 1979 (04) 287-289.

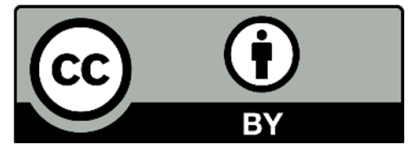

(C) 2021 by the authors; licensee Growing Science, Canada. This is an open access article distributed under the terms and conditions of the Creative Commons Attribution (CC-BY) license (http://creativecommons.org/licenses/by/4.0/). 